\title{
Endoscopic resection of undifferentiated-type gastric carcinoma: an extreme step to be restrained?
}

\author{
TAKeshi SANO \\ Department of Gastroenterological Surgery, Cancer Institute Hospital, 3-8-31 Ariake, Koto-ku, Tokyo 135-8550, Japan
}

Endoscopic mucosal resection (EMR) of gastric tumor was commenced in Japan in the 1970 s to remove small superficial adenocarcinomas. Lymph node metastasis was the only limiting factor for EMR to be a curative treatment, and a number of studies were conducted using the surgical databases to specify gastric lesions that had never metastasized to the lymph nodes. All studies consistently showed that at least small differentiated (D-type) adenocarcinomas confined to the mucosal layer without ulceration were free from nodal metastasis.

Because some safety margin was indispensable for a new procedure to be introduced in cancer therapy, the original indications of EMR were strictly limited to tiny tumors as described below. In the late 1990s an innovative new technique emerged using specially designed needle knives (endoscopic submucosal dissection, ESD) [1], which has dramatically changed the procedure of endoscopic resection. ESD enabled resection of large tumors or those having an ulcer component (more precisely, ESD was invented because of the necessity of resecting these tumors). Consequently, expansion of the original criteria was proposed [2] and has been adopted in specialized institutions for more than 10 years, showing satisfactory results in terms of patients' survival [3].

The original EMR criteria were composed of four primary factors: (a) depth of tumor invasion - mucosa; (b) histological type - D-type; (c) tumor size $-2 \mathrm{~cm}$; (d) ulcer component (breakdown or fibrosis of the muscularis mucosa) - none. The expanded criteria first relaxed conditions (c) and (d), then (a), finally advocating ESD for intramucosal cancer regardless of tumor size without an ulcer component, or intramucosal cancer less than $3 \mathrm{~cm}$ in size with an ulcer component, or

Offprint requests to: T. Sano minute submucosal invasive cancer less than $3 \mathrm{~cm}$, all on condition that the tumor is histologically of the differentiated type.

Criterion (b) was untouched for a long time because most studies failed to specify the category of undifferentiated type (UD-type) tumors free from nodal metastasis. UD-type adenocarcinomas mostly had an ulcer component and could metastasize regardless of size or depth. This does not mean that UD-type T1 tumors are oncologically more aggressive than D-type T1 tumors. The patients' survival is similar or even better in the UD-type as long as they are treated by gastrectomy with lymphadenectomy [4]. The potential of lymphatic invasion, however, seems higher in UD-type tumors, which makes ESD difficult.

A suggestion was made in 2000 that UD-type intramucosal cancer less than $2 \mathrm{~cm}$ in size without an ulcer component or lymphatic-vascular capillary involvement might be suitable for ESD [2]. However, such tumors were so rare that it was only in 2009 that the authors completed a collection of 310 such cases (of 3,834 T1 tumors of UD-type) treated by surgery, found no lymph node metastasis, and thereby concluded the risk is nil, with a very narrow $95 \%$ confidence interval, $0-0.96 \%$ [5].

It seemed that the final hurdle had been successfully crossed. Then two brief reports were submitted to this journal, each describing a case of lymph node metastasis from a UD-type tumor that satisfied the above criteria. The case reported by Nasu and colleagues in this issue [6] was a 13-mm IIc lesion with signet-ring cells spreading only in the superficial third of the mucosal layer. No ulcer, breakdown of muscularis mucosa, or submucosal fibrosis was seen. Five perigastric lymph nodes in the tumor drainage area contained signet-ring cell carcinoma. The second case, reported by Hirasawa and colleagues [7], in this issue as well, was also a 13-mm IIc lesion of poorly differentiated adenocarcinoma and signet-ring cell carcinoma. A large lymph node along 
the common hepatic artery had poorly differentiated adenocarcinoma, and this was the only metastasis among the 61 nodes dissected.

The latter case represented a problem inherent in the ESD criteria. When the authors' original manuscript was submitted to the journal, the tumor had satisfied the expanded criteria of ESD. Our reviewers requested additional immunohistochemical staining to confirm the absence of fibrosis and lymphovascular involvement, which the authors provided. During the procedure, they found a single signet-ring cell in a lymphatic capillary of the mucosal layer in a newly made section. This was the only event in more than 60 sections made for this $13-\mathrm{mm}$ tumor. The case now does not meet the ESD criteria in a strict sense, but routine examination of the sections at 2-mm intervals in an ESD specimen would easily overlook such an event.

In all previous studies to establish the ESD criteria, the examined materials were surgical specimens of which primary tumors were usually sectioned at $5-\mathrm{mm}$ intervals and lymph nodes were simply bisected. If the primary tumor sections had been thinner, lymphovascular involvement or deeper tumor invasion would have been diagnosed in some cases. Similarly, if the lymph nodes had been examined by multiple sections, metastasis would have been detected more often. The proposed ESD criteria, therefore, should not be regarded as the absolute conditions to rule out the possibility of lymph node metastasis. No effort should be spared to review and refine the criteria, because ESD may leave nodal metastasis that would eventually kill the patient who would have been cured by gastrectomy with lymphadenectomy. This possibility should be minimized.

The clinical significance of lymph node metastasis that ESD may leave is a different issue. The patient in the first case noted above had positive nodes and has been disease-free for 6 years after gastrectomy. If these nodes had been left in the abdomen, would they have grown and killed the patient? Japanese surgeons will immediately say yes, while some others may be skeptical. In order to prove EMR/ESD as effective as, or even better than, gastrectomy in terms of survival, a randomized study should be conducted. However, considering the fact that endoscopic resection has been in practice for more than 20 years without any oncological problems raised, and that EMR/ESD is undoubtedly superior to gastrectomy in terms of patients' quality of life, it is impossible to plan a randomized trial in Japan. Instead, some oncology groups are prospectively collecting the ESD data by expanded criteria to confirm its safety and efficacy in a phase II setting. Meanwhile, particular attention should be paid to the adoption of the expanded criteria for UD-type adenocarcinoma, knowing the possibility of exceptional cases such as those presented in this issue.

\section{References}

1. Ono H, Kondo H, Gotoda T, Shirao K, Yamaguchi H, Saito D, et al. Endoscopic mucosal resection for treatment of early gastric cancer. Gut 2001;48:225-9.

2. Gotoda T, Yanagisawa A, Sasako M, Ono H, Nakanishi Y, Shimoda $\mathrm{T}$, et al. Incidence of lymph node metastasis from early gastric cancer: estimation with a large number of cases at two large centers. Gastric Cancer 2000;3:219-25.

3. Gotoda T, Iwasaki M, Kusano C, Seewald S, Oda I. Endoscopic resection of early gastric cancer treated by guideline and expanded National Cancer Centre criteria. Br J Surg 2010;97:868-71.

4. Sano T, Sasako M, Kinoshita T, Maruyama K. Recurrence of early gastric cancer. Follow-up of 1475 patients and review of Japanese literature. Cancer 1993;72:3174-8.

5. Hirasawa T, Gotoda T, Miyata S, Kato Y, Shimoda T, Taniguchi H, et al. Incidence of lymph node metastasis and the feasibility of endoscopic resection for undifferentiated-type early gastric cancer. Gastric Cancer 2009;12:148-52.

6. Nasu J, Hori S, Asagi A, Nishina T, Ikeda Y, Tanimizu M, et al. A case of small undifferentiated intramucosal gastric cancer with lymph node metastasis. Gastric Cancer 2010;13:264-6.

7. Hirasawa T, Fujisaki J, Fukunaga T, Yamamoto Y, Yamaguchi T, Katori M, et al. A case of lymph node metastasis from undifferentiated type mucosal gastric cancer satisfying the expanded criteria for endoscopic resection based on routine histological examination. Gastric Cancer 2010;13:267-70. 